\title{
Plemelj Projection Operators over Domain Manifolds
}

\author{
John Ryan \\ Department of Mathematics, University of Arkansas, \\ Fayetteville, AR 72701, U. S. A.
}

July 9, 2021

\begin{abstract}
Plemelj projection operators are introduced for spaces of square integrable functions defined over the boundaries of a class of compact real n-dimensional manifolds lying in $C^{n}$. These manifolds posses many properties similar to domains in $R^{n}$, and are consequently called domain manifolds. The key ingredients used here are techniques from both real and complex Clifford analysis. Analogues of the Kerzman-Stein kernel and Szegö projection operators are introduced, and their conformal covariance is described.
\end{abstract}

\section{Introduction}

It is reasonably well known that given any reasonably smooth curve, $S$, lying in the complex plane and dividing $C$ into two open components, then $L^{p}(S)=H^{p}\left(S^{+}\right) \oplus H^{p}\left(S^{-}\right)$for $1<p<\infty$. Here $S^{+}$and $S^{-}$are the respective domains that complement the curve $S$, and $H^{p}\left(S^{ \pm}\right)$are the Hardy spaces of analytic functions defined on $S^{ \pm}$respectively and which extend continuously in the $L^{p}$ sense to their common boundary, $S$. This decomposition of $L^{p}(S)$ is obtained using Plemelj projection operators, which in turn are described using the singular Cauchy transform over $S$ and Cauchy's integral formula, see for instance $[\mathrm{B}]$. 
When turning to higher dimensions one can consider a sufficiently smooth, orientable surface, $S$, lying in $R^{n}$ and such that $S$ divides $R^{n}$ into two complementary domains, $S^{+}$and $S^{-}$respectively. Again one may consider the space $L^{p}(S)$ of $p$-integrable functions over $S$ where $1<p<\infty$. By introducing the Clifford algebra generated from $R^{n}$, the Dirac operator over $R^{n}$ the associated Cauchy integral formula and singular Cauchy transform over $S$ one can obtain the decomposition $L^{p}(S)=H^{p}\left(S^{+}\right) \oplus H^{p}\left(S^{-}\right)$. The spaces $H^{p}\left(S^{ \pm}\right)$are the spaces of solutions to the Dirac equation, or generalized Cauchy-Riemann system, which are defined on $S^{ \pm}$respectively and continuously extend in the $L^{p}$ sense to their common boundary, $S$. This decomposition is outlined for the cases where $S$ is compact and either $C^{2}$ or Liapunov in [GuSp1], and for the cases where $S$ is a Lipschitz graph in [Mi, LMcQ].

In all these cases one needs to assume that $L^{p}(S)$ is the space of Clifford algebra valued $p$ integrable functions defined on $S$. As the Clifford algebra contains a unit then the usual complex space of $L^{p}$ is contained in this space.

This decomposition has proved to be a fundamental result in Clifford analysis and has provided a fundamental link between it and the study of many types of boundary value problems. See for instance [Be, GuSp1, GuSp2, Mi, LMcQ].

In greater generality one may use operator theory associated to general Dirac operators and associated Clifford algebras to obtain similar results over compact, orientable, smooth submanifolds of codimension 1 lying within a smooth, orientable, Riemannian manifold. See for instance [B-BWo, C].

In separate work, [R1] and references therein, the author has introduced a class of smooth, orientable, real $n$-dimensional manifold which have many basic properties of domains lying in $R^{n}$, see also [GuKi, S]. Such manifolds are called domain manifolds. In [GuKi] it is shown that one can set up and solve boundary value problems over domain manifolds in much the same way as one does in complex analysis and Clifford analysis over Euclidean space. In particular one can set up Plemelj formulae, see [R1]. However, in [R1] these analogues of Plemelj formulae are introduced only for spaces of Hölder continuous functions defined on the boundary of a domain manifold, there referred to as a manifold of type one or two.

In this paper the intention is to introduce Plemelj projection operators and the analogous Hardy space decompositions over domain manifolds lying in both $C^{n}$. We also indicate how the results presented here extend to the 
analogues of domain manifolds lying in the complex sphere. Throughout we shall restrict attention to the cases where the boundary of the domain manifold is $C^{2}$.

\section{Preliminaries}

In this section we will develop the background on Clifford analysis that we shall need here.

We shall first consider the real, $2^{n}$ dimensional Clifford algebra, $C l_{n}$, generated from $R^{n}$. So if $R^{n}$ has orthonormal basis $e_{1}, \ldots, e_{n}$ then $C l_{n}$ is chosen so that it has basis $1, e_{1}, \ldots, e_{n}, \ldots, e_{j_{1}} \ldots e_{j_{r}}, \ldots, e_{1} \ldots e_{n}$, where $1 \leq$ $j_{1}<\ldots<j_{r} \leq n$ and $e_{i} e_{j}+e_{j} e_{i}=-2 \delta_{i, j}$ where $\delta_{i, j}$ is the Kroneker delta function. It may be seen that we are considering $R^{n}$ to be embedded in $C l_{n}$.

Although in general $C l_{n}$ is not a division algebra it may be seen that each non-zero vector $x \in R^{n}$ has a multiplicative inverse $x^{-1}=\frac{-x}{\|x\|^{2}}$. Up to a sign this inverse corresponds to the Kelvin inverse of a non-zero vector.

We shall need the following antiautomorphism over $C l_{n}$

$$
-: C l_{n} \rightarrow C l_{n}:-\left(e_{j_{1}} \ldots e_{j_{r}}\right)=(-1)^{r} e_{j_{r}} \ldots e_{j_{1}} .
$$

For $a=a_{0}+\ldots+a_{1, \ldots, n} e_{1} \ldots e_{n} \in C l_{n}$ we shall write $\bar{a}$ for $-a$. It may be noted that the real part of $a \bar{a}$ gives the square of the norm, $\|a\|$, of $a$, where $\|a\|=\left(a_{0}^{2}+\ldots+a_{1, \ldots, n}^{2}\right)^{\frac{1}{2}}$.

We shall also need the complexification $C l_{n}(C)$ of $C l_{n}$. The antiautomorphism, - extends linearly to this algebra. As is usual we shall denote the complexification of $R^{n}$ by $C^{n}$. It is no longer the case that each non-zero vector in $C^{n}$ has a multiplicative inverse. For instance we may consider $e_{1}+i e_{2}$. In this case $\left(e_{1}+i e_{2}\right)^{2}=0$. We shall denote a vector $z_{1} e_{1}+\ldots+z_{n} e_{n} \in C^{n}$ by $z$, and we shall denote the null cone $\left\{z \in C^{n}: z^{2}=0\right\}$ by $N(0)$. For $z_{1} \in C^{n}$ we denote the null cone $\left\{z \in C^{n}:\left(z-z_{1}\right)^{2}=0\right\}$ by $N\left(z_{1}\right)$. It may be noted that each vector $z \in C^{n} \backslash N(0)$ has a multiplicative inverse.

Definition 1 A smooth real $n$-dimensional manifold $M$ lying in $C^{n}$ is called a domain manifold if for each $z \in M$

(i) $N(z) \cap M=\{z\}$

(ii) $N(z) \cap T M_{z}=\{z\}$. 
Such manifolds have been used extensively in [GuKi, R1, S]. Here we shall assume that the boundary of each domain manifold considered is at worst $C^{2}$, though similar results to the ones presented here can be obtained with only minor modification to the techniques used here if one assumed that the boundary is $C^{1}$ and has Hölder continuous derivative with respect to some atlas. We shall not consider the case where the boundary of $M$ is Lipschitz. When the domain manifold is a subset of $R^{n}$ then its interior is a domain in $R^{n}$. It is for this reason that such manifolds are called domain manifolds.

Associated to each domain manifold $M$ is a domain in $C^{n}$ called a cell of harmonicity. This domain is the component of $C^{n} \backslash \cup_{z \in \partial M} N(z)$ which contains the interior of $M$. We shall denote this domain by $M^{\dagger}$. When $M$ is a domain in $R^{n}$ then $M^{\dagger}$ is the cell of harmonicity, or Vekua hull, described in $[\mathrm{A}]$ and elsewhere.

Definition 2 For $U$ a domain in $R^{n}$ a $C l_{n}(C)$ valued differentiable function $f(x)$ defined on $U$ is said to be left monogenic if $D f=0$ everywhere on $U$, where $D=\sum_{j=1}^{n} e_{j} \frac{\partial}{\partial x_{j}}$. Similarly a $C l_{n}(C)$ valued differentiable function $g$ defined on $U$ is said to be right monogenic if $g D=0$ everywhere on $U$, where $g D=\Sigma_{j=1}^{n} \frac{\partial g}{\partial x_{j}} e_{j}$.

The following two theorems are standard to Clifford analysis and can be found in [BDSo, GMu] and elsewhere.

Theorem 1 (Cauchy's Theorem) Suppose that $f$ and $g$ are respectively left and right monogenic functions on $U$ and that $S$ is a compact $C^{1}$ surface bounding a subdomain of $U$ then

$$
\int_{S} g(x) n(x) f(x) d \sigma(x)=0
$$

where $n(x)$ is the outward pointing normal vector to $S$ at $x$ and $\sigma$ is the usual Hausdorff measure on $S$.

Theorem 2 (Cauchy's Integral Formula) Suppose that $f$ is a left monogenic function on the domain $U$ and that $S$ is a compact $C^{1}$ surface bounding a subdomain $V$ of $U$. Then for each $y \in V$

$$
f(y)=\frac{1}{\omega_{n}} \int_{S} G(x-y) n(x) f(x) d \sigma(x)
$$


where $\omega_{n}$ is the surface area of the unit sphere in $R^{n}$ and $G(x)$ is the left and right monogenic function $\frac{x-y}{\|x-y\|^{n}}$.

If $U^{\dagger}$ is the cell of harmonicity associated to the domain $U$ then the previous theorem can be applied to show that when $n$ is even the left monogenic function has a unique holomorphic continuation to a function $f^{\dagger}$ defined on $U^{\dagger}$. Moreover $f^{\dagger}$ satisfies the the equation $D_{C} f^{\dagger}=0$ where $D_{C}=\sum_{j=1}^{n} e_{j} \frac{\partial}{\partial z_{j}}$. When $n$ is odd then $f^{\dagger}$ is defined on some Riemann surface covering $U^{\dagger}$.

Definition 3 Suppose that $\Omega$ is a domain in $C^{n}$ or a Riemann surface covering a domain in $C^{n}$ and $f(z)$ is a holomorphic function defined on $\Omega$ and takes values in $C l_{n}(C)$. Then if $f$ satisfies the equation $D_{C} f=0$ then $f$ is called a complex left monogenic function. Similarly if $g$ is a $C l_{n}(C)$ valued holomorphic function defined on $\Omega$ and satisfying $g D_{C}=0$ then $g$ is called a complex right monogenic function.

The following two theorems are treated in depth in $[R 1, S]$.

Theorem 3 Suppose that $f$ and $g$ are respectively complex left and right monogenic functions defined in a neighbourhood $\Omega$ of a compact domain manifold $M$. Then

$$
\int_{\partial M} g(z) W z f(z)=0
$$

where $W z$ is the holomorphic differential form $\sum_{j=1}^{n}(-1)^{n} e_{j} d z_{1} \wedge \ldots \wedge d z_{j-1} \wedge$ $d z_{j+1} \wedge \ldots \wedge d z_{n}$.

Theorem 4 Suppose that $n$ is even and that $f$ is a complex left monogenic function defined in a neighbourhood of a compact domain manifold $M$. Then $f$ has a unique holomorphic continuation to a complex left holomorphic function $f^{\dagger}$ on $M^{\dagger}$ and for each $w \in M^{\dagger}$

$$
f^{\dagger}(w)=\frac{1}{\omega_{n}} \int_{\partial M} G(z-w) W z f(z)
$$

where $G(z)=(-1)^{\frac{n}{2}} z^{-n+1}$ is the complex left and right monogenic extension of $G(x)$ to $C^{n} \backslash N(0)$. 
In the case where $n$ is odd the complex left monogenic function defined by Equation 2 is defined on some Riemann surface covering a domain containing $M$.

In $[\mathrm{QR}]$ it is pointed out that the differential form $W z$ appearing in equations 1 and 2 can be replaced so that equation 1 becomes

$$
\int_{\partial M} g(z) n(z) f(z) d \sigma(z)=0
$$

while equation 2 becomes

$$
f^{\dagger}(w)=\frac{1}{\omega_{n}} \int_{\partial M} g(w-z) n(z) f(z) d \sigma(z)
$$

where $n(z)$ is a vector in $C^{n}$ orthogonal, with respect to the inner product $<z, w\rangle=\sum_{j=1}^{n} z_{j} w_{j}$, to the complexification of the tangent space $T \partial M_{z}$. Moreover $\sigma$ is a complex valued measure on $\partial M$. In $[\mathrm{QR}]$ it is observed that the function $n(z)$ is non-zero and $C^{1}$.

\section{Hardy Spaces over Domain Manifolds}

Definition 4 Suppose that $M$ is a domain manifold then for $1<p<\infty$ a function $f: \partial M \rightarrow C l_{n}(C)$ is said to belong to the $L^{p}$ space, $L^{p}(\partial M)$, of $\partial M$ if

$$
\left(\int_{\partial M}\|f(z)\|^{p}|d \sigma(z)|\right)^{\frac{1}{p}}<\infty
$$

In the previous definition the term $|d \sigma(z)|$ stands for the infinitesimal of the absolute value measure $\sigma \mid$ of the complex measure $\sigma$ and it is introduced in $[\mathrm{QR}]$.

For each pair $f, g \in L^{2}(\partial M)$ there is a well defined inner product

$$
<f, g>_{\partial M}=\int_{\partial M} \overline{f(z)} g(z) d \sigma(z) .
$$

This integral is dominated by $\int_{\partial M} f(z)^{\star} g(z) d \sigma(z)$, where $f^{\star}$ is the complex conjugate of $\bar{f}$. The identity component of

$$
\int_{\partial M} f^{\star}(z) f(z)|d \sigma(z)|
$$


gives the square of the $L^{2}$ norm of $f$ for each $f \in L^{2}(\partial M)$.

When $M$ is a subset of $R^{n}$ this definition of an $L^{p}$ space corresponds to the usual definition of the $L^{p}$ space over the boundary of some domain in $R^{n}$.

The following result is an immediate consequence of theorem 2.6 on page 223 of $[\mathrm{StW}]$.

Proposition 1 Suppose that $V$ is a real $n-1$ dimensional vector subspace of $C^{n}$. Then for $1<p<\infty$ the integral

$$
P . V \cdot \frac{1}{\omega_{n}} \int_{V} G(z-w) n(w) f(w) d \sigma(w)
$$

defines a bounded linear operator

$$
C_{V}: L^{p}(V) \rightarrow L^{p}(V) .
$$

Using this result we can now deduce:

Theorem 5 Suppose that $M$ is a compact domain manifold then the integral

$$
P . V \cdot \frac{1}{\omega_{n}} \int_{\partial M} G(z-w) n(w) f(w) d \sigma(w)
$$

defines a bounded linear operator

$$
C_{\partial M}: L^{2}(\partial M) \rightarrow L^{2}(\partial M) .
$$

Proof: The proof follows a standard argument for compact, $C^{2}$, or even Liapunov, curves lying in the complex plane. This argument was adapted to the Clifford analysis setting for sufficiently smooth compact surfaces lying in $R^{n}$ in [Be]. The argument makes use of a cancellation property given by the $C^{1}$ function $n(w)$ over $\partial M$.

Essentially for each $z \in \partial M$ we may find an $\epsilon(z) \in R^{+}$and a $C^{1}$ homotopy

$$
H_{z}: B(z, \epsilon(z)) \cap \partial M \times[0,1] \rightarrow C^{n}
$$

such that

(i) $H(w, 0)=w$

(ii) $H(B(z, \epsilon(z) \cap \partial M), 1)=B(z, \epsilon(z)) \cap T \partial M_{z}$.

(iii) $\|H(w, 1)-z\|=\|w-z\|$.

Moreover, 
(iv) there is a $\delta(z) \in R^{+} \cup\{0\}$ such that $\|w-H(w, 1)\|<\delta(z)\|w-z\|$ for each $w \in B(z, \epsilon(z)) \cap \partial M$.

Now

$$
\begin{gathered}
\int_{B(z, \epsilon(z)) \cap \partial M} G(z-w) n(w) f(w) d \sigma(w)= \\
\int_{B(z, \epsilon(z)) \cap \partial M} G(z-w)(n(w)-n(z)) f(w) d \sigma(w) \\
+\int_{B(z, \epsilon(z)) \cap \partial M} G(z-w) n(z) f(w) d \sigma(w) .
\end{gathered}
$$

As $n(w)$ is a $C^{1}$ function over $\partial M$ then the first term on the right side of the previous equation defines a weakly singular integral operator acting on the square integrable function $f$.

We can now use the homotopy $H$ and Proposition 1 to deal with the term

$$
\int_{B(z, \epsilon(z)) \cap \partial M} G(z-w) n(z) f(w) d \sigma(w)
$$

This term can be rewritten as

$$
\begin{gathered}
\int_{B(z, \epsilon(z)) \cap \partial M}(G(z-w)-G(z-H(w, 1))) n(z) f(w) d \sigma(w) \\
+\int_{B(z, \epsilon(z)) \cap \partial T M_{z}} G(z-v) n(z) \lambda_{z}(v) f\left(\psi_{z}(v)\right) d \sigma(v),
\end{gathered}
$$

where $\psi_{z}$ is the $C^{1}$ diffeomorphism defined by $H\left(\psi_{z}(v), 1\right)=v$, and $\lambda_{z}$ is the Jacobian associated to the $C^{1}$ function $\psi_{z}$.

By conditions (iii) and (iv) of the homotopy $H$ the first term on the right side of the previous equation is dominated by the term

$$
C \delta(z) \int_{\left.B_{(} z, \epsilon(z)\right) \cap \partial M}\|z-w\|^{-n+2}\|n(z) f(w)\||d \sigma(w)|
$$

for some dimensional constant $C \in R^{+}$, and so again defines a weakly singular integral operator acting on $f$.

As $\lambda_{z}$ is a bounded measurable function it follows from proposition 1 that the second term on the right side of the same equation also defines an $L^{2}$ bounded operator.

Moreover, the term

$$
\int_{\partial M \backslash(B(z, \epsilon(z)) \cap \partial M)} G(z-w) n(w) f(w) d \sigma(w)
$$


is $L^{2}$ bounded.

So far we have shown that for each $z \in \partial M$ the operator

$$
T_{z}: L^{2}(\partial M) \rightarrow L^{2}(\partial M)
$$

defined by

$$
\int_{\partial M \cap B(z, \epsilon(z))} G(z-w) n(w) f(w) d \sigma(w)
$$

for each $f \in L^{2}(\partial M)$, is an $L^{2}$ bounded operator. The result now follows from the compactness of $\partial M$.

If in Proposition 1 and Theorem 5 we replace the kernel $G(z)$ by a kernel $K(z)=\frac{\Omega(z)}{\left(z^{2}\right)^{\frac{n-2}{2}}}$ where $\Omega(z)$ is an odd function homogeneous of degree zero, we also obtain an $L^{2}$ bounded operator

$$
T_{K}: L^{2}(\partial M) \rightarrow L^{2}(\partial M): T_{K}(g)(w)=P . V . \int_{\partial M} K(w-z) n(z) g(z) d \sigma(z) .
$$

The proof is the same as the proof of Theorem 5 .

Suppose that $g$ belongs to the function space $C^{1}(\partial M)$ of $C l_{n}(C)$ valued $C^{1}$ functions on $\partial M$. Suppose also that $\theta:[0,1) \rightarrow M$ is piecewise smooth with $\lim _{t \rightarrow 1} \theta(t)=w \in \partial M$ and $\theta^{\prime}(1)$ does not belong to $T M_{w}(C)$, the complexification of the tangent space $T M_{w}$. Then in [R1] we show that

$$
\begin{gathered}
\lim _{t \rightarrow 1} \frac{1}{\omega_{n}} \int_{\partial M} G(\theta(t)-z) n(z) g(z) d \sigma(z)= \\
\frac{1}{2} g(w)+\frac{1}{\omega_{n}} P . V \cdot \int_{\partial M} G(w-z) n(z) g(z) d \sigma(z) .
\end{gathered}
$$

Using the fact that $C^{1}(\partial M)$ is a dense subspace of $L^{2}(\partial M)$ we immediately have:

Theorem 6 Suppose that $f \in L^{2}(\partial M)$ and that $\theta$ and $w$ are as in the preceding paragraph. Then

$$
\begin{gathered}
\lim _{t \rightarrow 1} \frac{1}{\omega_{n}} \int_{\partial M} G(\theta(t)-z) n(z) f(z) d \sigma(z)= \\
\frac{1}{2} f(w)+\frac{1}{\omega_{n}} P . V . \int_{\partial M} G(w-z) n(z) f(z) d \sigma(z)
\end{gathered}
$$


This last formula is a generalization of the Plemelj formula arising in one variable complex analysis.

We would like to show that the pointwise convergence described in the previous equation can be replaced by uniform convergence in the $L^{2}$ norm. To this end we first introduce maximal functions.

Definition 5 Suppose that $f \in L^{p}(\partial M)$ and $1<p<\infty$ then for each $w \in \partial M$ we define the maximal function $M(f)(w)$ of $f(w)$ to be

$$
\sup _{r>0} \frac{1}{\int_{B(w, r) \cap \partial M}|d \sigma(z)|} \int_{B(w, r) \cap \partial M}\|f(z)\||d \sigma(z)| .
$$

By similar arguments to those presented in [St] it may be deduced that for each $f \in L^{p}(\partial M)$ and with $1<p<\infty$ then $M(f) \in L^{p}(\partial M)$ and there is a positive constant $C(n, p)$ such that

$$
\|M(f)\|_{L^{p}} \leq C(n, p)\|f\|_{L^{p}} .
$$

We also will need non-tangential maximal functions. By identifying $C^{n}$ with $R^{2 n}$ endowed with the usual inner product $<,>_{R^{2 n}}$ for each $w \in C^{n}$ each $u \in C^{n} \backslash\{0\}$ and each $\alpha \in\left(0, \frac{\pi}{2}\right)$ we can introduce the cone $\Gamma(w, u, \alpha)=$ $\left\{z \in C^{n}: 0<<z-w, u>_{R^{2 n}}<\|z-w\| \cos \alpha\right\}$. For each $r \in R^{+}$we define the truncated cone $\Gamma(w, u, \alpha, r)$ to be the set $\{z \in \Gamma(w, u, \alpha):\|z-w\|<r\}$. Both the sets $\Gamma(w, u, \alpha)$ and $\Gamma(w, u, \alpha, r)$ are open subsets of $C^{n}$.

As the manifold $M$ is compact and has $C^{2}$ boundary then we may find an $\alpha(M) \in\left(0, \frac{\pi}{2}\right)$ and an $r(M) \in R^{+}$such that $\Gamma(w, n(w), \alpha(M), r(M)) \subset M^{\dagger}$ and $\partial \Gamma(w, n(w), \alpha(M), r(M)) \cap \partial M^{\dagger}=\{w\}$. for each $w \in \partial M$.

Definition 6 For each $p \in(0, \infty)$ and each $f \in L^{p}(\partial M)$ we define the nontangential maximal function $N(f)(w)$ of $f$ to be

$$
\sup _{z \in \Gamma(w, n(w), \alpha(M), r(M))}\left\|\int_{\partial M} G(z-u) n(u) f(u) d \sigma(u)\right\|
$$

The non-tangential maximal function introduced in the previous definition differs from the usual one set up over domains in $R^{n}$. There one considers supremums over real n-dimensional cones lying in $R^{n}$. Here we have doubled the dimension and are taking supremums over complex, n-dimensional cones in $C^{n}$. 
Theorem 7 Suppose that $f \in L^{2}(\partial M)$ then $\|N(f)\|_{L^{2}}<C(M)\|f\|_{L^{2}}$ for some $C(M) \in R^{+}$.

Proof: The proof is an adaptation of arguments appearing on page 63 of [Mi] and page 27 of [MCo].

Let us begin by considering

$$
\int_{\partial M \backslash(B(w, \epsilon) \cap \partial M} G(w-z) n(z) f(z) d \sigma(z)-\int_{\partial M} G(u-z) n(z) f(z) d \sigma(z),
$$

where $w \in \partial M$ and $u \in M^{\dagger}$. This expression is equal to

$$
\begin{gathered}
\int_{\partial M \backslash(B(w, \epsilon) \cap \partial M}(G(w-z)-G(u-z)) n(z) f(z) d \sigma(z) \\
-\int_{B(w, \epsilon) \cap \partial M} G(u-z) n(z) f(z) d \sigma(z) .
\end{gathered}
$$

Let us restrict $u$ so that $\|w-u\|=\epsilon$ and $u \in \Gamma(w, n(w), \alpha(M), r(M))$. Now

$$
\begin{gathered}
\left\|\int_{B(w, \epsilon) \cap \partial M} G(u-z) n(z) f(z) d \sigma(z)\right\| \leq C \frac{1}{\epsilon^{n-1}} \int_{B(w, \epsilon) \cap \partial M}\|f(z)\||d \sigma(z)| \\
\leq C_{1} M(f)(w),
\end{gathered}
$$

for some $C$ and $C_{1} \in R^{+}$.

Moreover

$$
\|G(w-z)-G(u-z)\| \leq C_{2} \frac{\epsilon}{\|z-w\|^{n}}
$$

for each $z \in \partial M \backslash\left(B(w, \epsilon) \cap \partial M\right.$ and some constant $C_{2} \in R^{+}$.

It follows that

$$
\begin{gathered}
\left\|\int_{\partial M \backslash(B(w, \epsilon) \cap \partial M)}(G(w-z)-G(u-z)) n(z) f(z) d \sigma(z)\right\| \leq \\
C_{2} \epsilon \sum_{j=0}^{\infty} \| \int_{\partial M \cap\left(B\left(w, 2^{j} \epsilon\right) \backslash B\left(w, 2^{j+1} \epsilon\right)\right)} \frac{\|f(z)\|}{\|z-w\|^{n}}|d \sigma(z)|,
\end{gathered}
$$

for some $C_{2} \in R^{+}$. The right side of this expression is dominated by $C_{3} M(f)(w)$ for some $C_{3} \in R^{+}$.

Consequently

$$
N(f)(w)<C_{4}\left(M(f)(w)+\sup _{\epsilon>0}\left\|\int_{B(w, \epsilon) \cap \partial M} G(w-z) n(z) f(z) d \sigma(z)\right\|\right),
$$


for some $C_{4} \in R^{+}$. This inequality is derived in the euclidean setting using much the same arguments in [Mi].

Now

$$
\begin{aligned}
\| \int_{\partial M \backslash(B(w, \epsilon) \cap \partial M)} & G(w-z) n(z) f(z) d \sigma(z) \| \leq\left(\left\|\int_{\partial M} G(w-z) n(z) f(z) d \sigma(z)\right\|\right. \\
& +\left\|\int_{B(w, \epsilon) \cap \partial M} G(w-z) n(z) f(z) d \sigma(z)\right\| .
\end{aligned}
$$

One can now readily adapt the proof of Cotlar's inequality given in [MCo] and show that

$$
\begin{gathered}
\sup _{\epsilon>0}\left\|\int_{\partial M \backslash B(w, \epsilon)} G(w-z) n(z) f(z) d \sigma(z)\right\| \leq \\
C\left(M\left(P . V . \int_{\partial M} G(w-z) n(z) f(z) d \sigma(z)\right)+M(f)(w)\right)
\end{gathered}
$$

for some constant $C \in R^{+}$.

The result now follows from Theorem 5 and Inequality 3 .

Combining Theorems 6 and 7 with Lebesgue's dominated convergence theorem we get:

Theorem 8 Suppose that $f \in L^{2}(\partial M)$ then for any smooth homotopy deformation $H: \partial M \times[0,1) \rightarrow M^{\dagger}$ such that $\lim _{t \rightarrow 1} H(w, t)=w$ for each $w \in \partial M$

$$
\begin{gathered}
\lim _{t \rightarrow 1} \| \frac{1}{\omega_{n}} \int_{\partial M} G(H(w, t)-z) n(z) f(z) d \sigma(z) \\
-\frac{1}{2} f(w)-\frac{1}{\omega_{n}} P . V . \int_{\partial M} G(w-z) n(z) f(z) \|_{L^{2}}=0 .
\end{gathered}
$$

Even in the special cases where $M$ is an open subset $U$ of $R^{n}$ Theorem 8 is a stronger statement than the usual statement of uniform convergence in the $L^{2}$ norm. This is because one usually looks for uniform convergence within the domain $U$ and not on the cell of harmonicity $U^{\dagger}$ associated to $U$.

Let us denote the $L^{2}$ bounded operator defined by

$$
\frac{1}{2} f(w)+\frac{1}{\omega_{n}} \int_{\partial M} G(w-z) n(z) f(z) d \sigma(z)
$$

by $S^{+}$and the singular integral operator $S^{+}-\frac{1}{2} I$ by $C_{\partial M}$. For each $f \in$ $L^{2}(\partial M)$ let us denote $S^{+} f$ by $f^{+}$. A simple application of Cauchy's integral 
formula and Theorem 6 reveal that $S^{+2}=S^{+}$and $C_{\partial M}^{2}=\frac{1}{4} I$. If we denote the $L^{2}$ bounded operator $\frac{1}{2} I-C_{M}$ by $S^{-}$then we can readily deduce that $S^{-2}=S^{-}$and $S^{+} S^{-}=S^{-} S^{+}=0$. This generalizes to $L^{2}(\partial M)$ results known to hold over the $L^{2}$ space of a closed reasonably smooth curve in the complex plane and for $L^{2}$ spaces of sufficiently smooth surfaces lying in $R^{n}$. See for instance [B, LMcQ] and elsewhere.

In [R1] we note that for any compact domain manifold $M$ we can construct another domain manifold $M^{\prime}$ such that $M \subset M^{\prime}$ and $\partial M$ is contained in the interior of $M^{\prime}$. Let us suppose that $\partial M$ is connected then let us denote the component of $C^{n} \backslash \cup_{z \in \partial M} N(z)$ containing $M^{\prime} \backslash c l(M)$ by $M^{\dagger^{\prime}}$, where $\operatorname{cl}(M)$ is the closure of $M$. By arguments described in [R1] it may be noted that $M^{\dagger^{\prime}}$ is a domain in $C^{n}$. In the cases where $\partial M$ is multiply connected then $M^{\dagger^{\prime}}$ is a union of finitely many disjoint domains.

By similar arguments to those used to deduce Theorem 6 one may deduce:

Theorem 9 Suppose that $f \in L^{2}(\partial M)$ then

$$
\lim _{u \rightarrow w} \frac{1}{\omega_{n}} \int_{\partial M} G(u-z) n(z) f(z) d \sigma(z)=-S^{-} f(w) \quad \text { a.e. }
$$

where $u \in M^{\dagger^{\prime}}, w \in \partial M$ and $u$ approaches $w$ non-tangentially.

If we denote $-S^{-} f$ by $f^{-}$for each $f \in L^{2}(\partial M)$, then $f=f^{+}+f^{-}$.

By similar arguments to those used to deduce Theorem 8 we can also derive:

Theorem 10 Suppose that $H: \partial M \times[0,1) \rightarrow M^{\dagger^{\prime}}$ is a smooth homotopy such that $\lim _{t \rightarrow 1} H(w, t)=w$ then

$$
\lim _{t \rightarrow 1}\left\|\frac{1}{\omega_{n}} \int_{\partial M} G(H(w, t)-z) n(z) f(z) d \sigma(z)-f^{-}(w)\right\|_{L^{2}}=0
$$

for each $f \in L^{2}(\partial M)$.

Definition 7 We shall call the space $S^{+} L^{2}(\partial M)$ the Hardy 2-space of $M^{\dagger}$ and we shall denote it by $H^{2}\left(M^{\dagger}\right)$.

Similarly we shall call the space $S^{-} L^{2}(\partial M)$ the Hardy 2-space of $M^{\dagger^{\prime}}$. We shall denote this space by $H^{2}\left(M^{\dagger^{\prime}}\right)$. 


\section{Theorem 11}

$$
L^{2}(\partial M)=H^{2}\left(M^{\dagger}\right) \oplus H^{2}\left(M^{\dagger^{\prime}}\right)
$$

Let $P^{+}: L^{2}(\partial M) \rightarrow H^{2}\left(M^{\dagger}\right)$ be the Szegö, or orthogonal, projection with respect to the inner product $<,>_{\partial M}$. Also let $P^{-}: L^{2}(\partial M) \rightarrow H^{2}\left(M^{\dagger^{\prime}}\right)$ be the Szegö, or orthogonal, projection with respect to the same inner product $<,>_{\partial M}$. These operators are self adjoint with respect to this inner product. In other words $P^{ \pm \star}=P^{ \pm}$where $P^{ \pm \star}$ is the adjoint of $P^{ \pm}$with respect to $<,>_{\partial M}$.

Also let $C_{\partial M}^{\star}(f)(w)=\frac{1}{\omega_{n}} n(w) \int_{\partial M} G(w-z) f(z) d \sigma(z)$. Then the $L^{2}$ bounded operator $C_{\partial M}-C_{\partial M}^{\star}$ is called the Kerzman-Stein kernel. This is in complete analogy to the complex variable setting, see [B].

We may easily obtain the following identities.

$P^{ \pm}=S^{ \pm} P^{ \pm}, P^{ \pm \star}=P^{ \pm \star} S^{ \pm \star}=P^{ \pm} S^{ \pm \star}$, and $S^{ \pm}=P^{ \pm} S^{ \pm}$. Consequently $P^{ \pm}-S^{ \pm}=P^{ \pm}\left(C_{\partial M}^{\star}-C_{\partial M}\right)$.

Hence

$$
P^{ \pm}\left(I-\left(C_{\partial M}^{\star}-C_{\partial M}\right)\right)=S^{ \pm} .
$$

These identities are generalizations of identities appearing in [B, Se].

As in the classical case, see [KSt], the singularities of the integral operators $C_{\partial M}$ and $C_{\partial M}^{\star}$ are cancelled out in $C_{\partial M}-C_{\partial M}^{\star}$. Consequently this Kerzman Stein kernel is a compact operator.

In order to move from the setting where $\partial M$ is compact to the more general setting we shall use Möbius transformations. In [Bo] and elswhere it is shown that the space of left monogenic functions over some domain remain invariant under Möbius transformations. Also in [QR] it is noted that cells of harmonicity transform to other cells of harmonicity under Möbius transformations. So in particular if $u=\psi(z)=(z+a)^{-1}$ for some constant $a \in C^{n}$ and $f(u)$ is a complex left monogenic function defined on a cell of harmonicity $M^{\dagger}$ then $G(z+a) f\left((z+a)^{-1}\right)$ is a complex left monogenic function on the cell of harmonicity $\psi^{-1}\left(M^{\dagger}\right)$. Also, [QR], $g(u) \in L^{2}(\partial M)$ if and only if $G(z+a) g\left((z+a)^{-1}\right) \in L^{2}\left(\partial \psi^{-1}(M)\right)$. Moreover, [QR], $\| G(z+$ a) $g\left((z+a)^{-1}\right)\left\|_{L^{2}}=\right\| g(u) \|_{L^{2}}$. It follows that $\left\|M\left(G(z+a) g\left((z+a)^{-1}\right)\right)\right\|_{L^{2}} \leq$ $\|g(u)\|_{L^{2}}$ for each $g \in L^{2}(\partial M)$.

In the case where $-a \in \partial M$ then $\partial M$ is no longer compact. Even though $\partial M$ is no longer compact its $C^{2}$ structure is preserved. 
In [QR] it is noted that if $v=(w+a)^{-1}$ then $G(v-u)=G(w)^{-1} G(w-$ $z) G(z)^{-1}$ and if $f, g \in L^{2}(\partial M)$ then

$$
\begin{gathered}
\int_{\partial M} f(u) n(u) g(u) d \sigma(u)= \\
\int_{\partial \psi^{-1}(M)} f\left((z+a)^{-1}\right) G(z+a) n(z) G(z+a) g\left((z+a)^{-1}\right) d \sigma(z) .
\end{gathered}
$$

From these remarks it is an easy exercise to transpose all results so far obtained here for the $L^{2}$ space of a compact $C^{2}$ manifold $\partial M$ to the setting where $\partial M$ is no longer compact but is the image under a Möbius transformation $\psi$ of a compact $C^{2}$ boundary of a domain manifold.

In [R2] we use a Cayley transformation to show that much of Clifford analysis over $R^{n}$ can also be set up over the sphere $S^{n}$ lying in $R^{n+1}$. Using this transformation it is a reasonably easy exercise to transpose the results presented here over domain manifolds and cells of harmonicity to their images

under this Cayley transformation within the complex sphere $S_{C}^{n}=\{z \in$ $\left.C^{n+1}: z^{2}=-1\right\}$.

\section{References}

[A] V. Avanissian, Cellule D'Harmonicité et Prolongement Analytique Complexe, Hermann, Paris, 1985.

[B] S. Bell, The Cauchy Transform, Potential Theory and Conformal Mapping, CRC Press, Boca Raton, 1992.

[Be] S. Bernstein, The left-linear Riemann problem in Clifford analysis, Bull. Belg. Math. Soc., 2, 1996, 557-576.

[Bo] B. Bojarski, Conformally covariant differential operators, Proceedings, XXth Iranian Math. Congress, Tehran, 1989.

[B-BWo] B. Booss-Bavnbeck and K. Wojciechowski Elliptic Boundary Problems for Dirac Operators, Birkhaüser, Basel, 1993.

[BDSo] F. Brackx, R. Delanghe and F. Sommen, Clifford Analysis, Pitman Research Notes in Mathematics, No, 76, London, 1982.

[C] D. Calderbank, Clifford analysis for Dirac operators of manifolds with boundary, to appear.

[GMo] J. Gilbert and M. A. M. Murray, Clifford Algebras and Dirac Operators in Harmonic Analysis, CUP, Cambridge, 1991.

[GuKi] K. Gürlebeck and F. Kippig, Complex Clifford analysis and elliptic 
boundary value problems, Advances in Applied Clifford Algebra, 5, 1995, 5162.

[GuSp1] K. Gürlebeck and W. Sprössig, Quaternionic Analysis and Elliptic Boundary Value Problems, Birkhaüser Verlag, Basel, 1990.

[GuSp2] K. Gürlebeck and W. Sprössig, Quaternionic and Clifford Calculus for Physicists and Engineers, Wiley and Sons, New York, 1997.

[LMcQ] C. Li, A. McIntosh and T. Qian, Clifford algebras, Fourier transforms and singular convolution operators on Lipschitz surfaces, Rev. Mat. Iberoamericana, 10, 1994, 665-721.

[KSt] N. Kerzman and E. M. Stein, The Cauchy kernel, the Szegö kernel, and the Riemann mapping function, Math. Ann., 236, 1978, 85-93.

[MCo] Y. Meyer and R. Coifman, Wavelets, Calderón-Zygmund and Multilinear Operators, CUP, Cambridge, 1997.

[Mi] M. Mitrea, Singular Integrals, Hardy Spaces, and Clifford Wavelets, Lecture Notes in Mathematics, No 1575, Springer Verlag, Heidelberg, 1994.

[QR] T. Qian and J. Ryan, Conformal transformations and Hardy spaces arising in Clifford analysis, Journal of Operator Theory, 35, 1996, 349-372.

[R1] J. Ryan, Plemelj formulae and transformations associated to plane wave decompositions in complex Clifford analysis, Proceedings of the London

Mathematical Society, 64, 1992, 70-94.

[R2] J. Ryan, Dirac operators on spheres and hyperbolae, Bolletin de la Sociedad Matematica a Mexicana, 3, 1996, 255-270.

[S] K. Sano, Another type of Cauchy's integral formula in complex Clifford analysis, Tokyo Journal of Mathematics, 20, 1997, 187-204.

[Se] S. Semmes, Chord-arc surfaces with small constant, 1, Advances in Mathematics, 85, 1991, 198-223.

[StW] E. M. Stein and G. Weiss, Introduction to Fourier Analysis on Euclidean Space, Princeton University Press, Princeton, 1971.

[St] E. M. Stein, Singular Integrals and Differentiability Properties of Functions, Princeton University Press, Princeton, 1970. 